\title{
DGMM - Informationen der MWE
}

Manuelle Medizin 2016 · 54:72

DOI 10.1007/s00337-015-0094-3

(c) Springer-Verlag

Berlin Heidelberg 2016

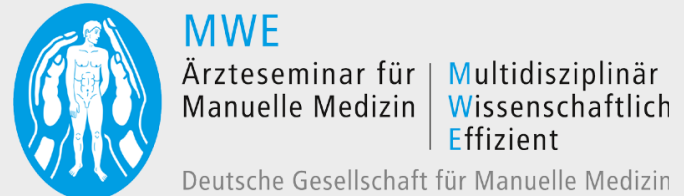

\section{Dr. Karl-Sell-Ärzteseminar Neutrauchburg (MWE) e. V.}

1. Vorsitzender:

Dr. med. Wolfgang von Heymann
Riedstr. 5, 88316 Isny

Tel. 07562 / 97 18-0

Fax $07562 / 971822$ info@manuelle-mwe.de www.manuelle-mwe.de

\section{Die MWE bietet für Ärzte:}

\section{Ein modernes wissenschaftliches Behandlungskonzept}

Die Manuelle Medizin hat sich - durch unser jahrelanges Engagement - zu einer sanften sehr effizienten medizinischen Heilmethode entwickelt, die bei einer großen Anzahl verschiedenster Beschwerden am menschlichen Bewegungsorgan erfolgreich eingesetzt werden kann. Sie ist frei von schädlichen Nebenwirkungen und steht heute in der Schmerztherapie - als fester Bestandteil modularer Behandlungskonzepte - auf einem festen wissenschaftlichen Fundament.

\section{Diagnosesicherheit}

In 320 Unterrichtseinheiten erfahren die Absolventen unserer Grundund Aufbaukurse den Körper als funktionale Einheit. Mit ihrem Tastsinn „begreifen“ sie Störungen und Blockaden am menschlichen Bewegungsorgan und erhalten einen Überblick über erfolgreiche Therapieansätze.

\section{Aktives Teaching}

Über 2/3 der gesamten Ausbildung besteht aus aktivem Teaching am Kollegen oder der Kollegin sowie am Patienten im Abschluss-/Prüfungskurs. Dadurch trainieren wir systematisch das pathogenetisch orientierte Denken.

\section{Standorte in ganz Deutschland}

MWE ist auch in Ihrer Nähe. Als größtes Seminar in Europa betreibt die MWE Ausbildungsstätten in ganz Deutschland. Sie finden uns in allen 16 Bundesländern und in Teilen Österreichs. Die Kurse sind überall einheitlich.

\section{Zeitnahe Erfolge in der Praxis}

Die Lehrinhalte sind mit ärztlicher Vorbildung leicht verständlich und nachvollziehbar. So ist es möglich, schon nach kurzer Zeit Behandlungserfolge in der eigenen Praxis zu erleben.

\section{On Top - Osteopathie für Ärzte}

Das Ausbildungskonzept der MWE gibt der Osteopathie einen festen Platz.

\section{Lernen nach amerikanischem Original}

Die Lehre von der Osteopathie für Ärzte orientiert sich am amerikanischen Original. In 390 Stunden lehren international anerkannte Dozenten des renommierten Philadelphia College of Osteopathic Medicine (PCOM) „das Hinhören und Behandeln mit den Händen“.

\section{Weiterbildung Manuelle Medizin - Einsteigerkurse}

\section{Grundkurs 1}

05.03.- 12.03 .16

10.06. -12.06 .16$

17.06.-19.06.16

24.06. -26.06 .16$

01.07.-03.07.16

Isny
Neuss
Neuss
Nürnberg
Nürnberg

Teil 1

Teil 2

Teil 1

Teil 2

Tagesrefresher

09.04.16

Damp Best of WS und Extremitätem

Wochenendrefresher

07.05.-08.05.16 Isny Obere Verkettung (HWS/Thorax)

\section{Gesamtrefresher/Kompetenzerhaltungskurs}

30.07.-06.08.16 Königstein

\section{Manuelle Medizin bei Kindern}

Kursort: Isny

Kursleiter: Dr. W. Coenen, Dr. M. Habring, Dr. A. Sammer, Dr. M. Riedel, Dr. P. Mantl

16.03.-19.03.16 Isny Kurs B Diagnostikkurs 2

Manualmedizin meets Wissenschaft

Behandlung manualmedizinischer Praxisbeispiele auf dem Boden der neurophysiologischen Grundlagen des Schmerzes

09.06.-12.06.16 Castelletto di Brenzone/Italien

Kinesio-Sport-Taping

22.04.-23.04.16 Isny

Neurale Strukturen

15.04.-16.04.16 Isny 